\title{
A BINARY LEVEL SET METHOD BASED ON K-MEANS FOR CONTOUR TRACKING ON SKIN CANCER IMAGES
}

\author{
Azadeh Noori Hoshyar, Adel Ali Al-Jumaily, Yee Mon Aung \\ University of Technology Sydney, Broadway 2007, Australia \\ Azadeh.NooriHoshyar@student.uts.edu.au,Adel.Al-Jumaily@uts.edu.au, Yee.M.Aung@ student.uts.edu.au
}

\begin{abstract}
A great challenge of research and development activities have recently highlighted in segmenting of the skin cancer images. This paper presents a novel algorithm to improve the segmentation results of level set algorithm with skin cancer images. The major contribution of presented algorithm is to simplify skin cancer images for the computer aided object analysis without loss of significant information and to decrease the required computational cost. The presented algorithm uses k-means clustering technique and explores primitive segmentation to get initial label estimation for level set algorithm. The proposed segmentation method provides better segmentation results as compared to standard level set segmentation technique and modified fuzzy cmeans clustering technique.
\end{abstract}

\section{KEY WORDS}

Segmentation, Clustering, Level set, K-means, Skin cancer.

\section{Introduction}

Segmentation is an essential issue in digital image processing and is used both for image description and as an important step before classification. The segmentation algorithms are generally based on similarity, which can be categorized as; thresholding [1], template matching [2], [3], region growing [4], edge detection [5], and clustering [6].

Clustering is a process of classifying a set of objects into classes with similar characteristics. It has been widely applied in many areas such as image processing [7], [8], machine learning [9],[10], pattern recognition [11]-[13], data mining [14]-[16], statistics [17], [18]. Recently clustering algorithms found crucial applications in medical imaging field [19].

Among clustering algorithms, k-means is the most popular one due to its simplicity and fast running speed. K-means is known as a simple and fast numerical, non-deterministic, unsupervised and iterative method which has proved to provide good clustering results [20]-[23].

Level set method is another powerful and robust segmentation technique which is flexible under challenging conditions. It depends on both extrinsic and intrinsic factors such as intensity and curvature, respectively [24][25]. Different researchers [26][27]indicated that level set method may decrease the mutability of complex segmentation tasks in medical applications. They mentioned the flexibility of level set techniques cause to lengthen computation time and consequently will limit its application in medical area. In some other works [28][29],the level set algorithm has been proposed to not be used merely for classification purposes.

In traditional level set methods, initialization extensively plays an essential role in curve evolution process. Reinitialization of level set function is significantly used as a numerical remedy to keep the curve evolution more stable and achieve effective results. Though, as pointed out by Gomes, many presented re-initialization schemes provide undesirable results along with increased computational cost [30]. On the other hand, it is becoming significantly obvious that none of the methods alone are adequate and that the application of different approaches will clear various aspects of data to be explored [31]-[34]. Thus, this work attempts to incorporate the strength of two techniques and overcome the disadvantages. Here, an implicit joint k-means-level set algorithm for classifier decision boundaries is presented. It applies k-means algorithm to get initial label estimation for level-set algorithm. In short, the contribution of this work is to propose a new and creative segmentation algorithm for development of the medical expert systems for achieving increased precision in diagnosis. The algorithm increases the accuracy of segmentation while decrease the computational cost.

The performance of proposed algorithm of level set classification is tested and compared using a data set comprising of forty skin cancer images. The parameters for performance evaluation include False positive error, Hammoude distance, True detection rate and Similarity. The results showed that the proposed approach is quite competitive with many classifiers that are being employed in practice.

The rest of this paper is structured as follows: We shortly go over the k-means framework in Section 2. In Section 3, the proposed algorithm is explained in detail. Experiment results are demonstrated in Section 4, and Section 5 is assigned to conclusion.

\section{The Basic K-means}

$\mathrm{K}$-means algorithm is a clustering algorithm which firstly presented by MacQueen in 1967[35]. This algorithm divides the pixels into $\mathrm{k}$ clusters and heavily relies on 
selecting the number of clusters $\mathrm{k}$ and initial cluster centroids vi, $\mathrm{i}=1, \ldots 2, \mathrm{k}$. The centroids of clusters are calculated based on the average of pixel intensities in each cluster. These initial centers effectively influence on number of iterations in k-means algorithm. After calculating the centroids $v_{i}$, the distance of pixels and centres are estimated and each pixel $\mathrm{x}_{\mathrm{j}}$ is iteratively assigned to the closest cluster as in equation 1 .

$$
\mathrm{d}_{\mathrm{ij}}=\left\|\mathrm{x}_{\mathrm{j}}-\mathrm{v}_{\mathrm{i}}\right\|
$$

The matrix of $U$ with the membership values are determined by

$$
\mathrm{U}=\left|\mathrm{u}_{\mathrm{ij}}\right|
$$

Where $u_{i j} \in\{0,1\}$ for all $i$ and $j . \sum_{t}^{k}=1 u_{i j}=1$ for all $j$

and $0<\sum_{j=1}^{n} u_{i j}<n$ for all $i$. $(k=$ number of clusters, $\mathrm{t}=0$,

$\mathrm{n}=$ number of pixels)

The cluster centres are updated by computing the mean of each cluster as in equation 3 .

$$
V_{i}=\frac{\sum_{j=1}^{n} u_{i j} x_{j}}{\sum_{j=1}^{n} u_{i j}} \text { for all } i
$$

This process is repeated till it reaches the center values same as the prior values since it indicates the current values are the optimal results.

This algorithm optimize the objective function $\mathrm{J}_{\mathrm{w}}(\mathrm{U}, \mathrm{v})$ in a manner that $k n$

$$
J_{W}(U, V)=\sum_{i=1}^{k} \sum_{j=1}^{n}\left\|X_{j}-V_{i}\right\|^{2}
$$

It is spotted that the algorithm is sensitive to cluster initialization and distance measure [19][20][36].

\section{The Level Set Framework}

Level set algorithms, firstly presented by Osher and Sethian [37, 38], offer an impressive implementation of curve evolution. This approach is based on enchasing contour $\mathrm{C}$ as the zero level set of the graph of a higher dimensional function $\varnothing(\mathrm{x}, \mathrm{y}, \mathrm{k})$, as in equation 5

$$
\mathrm{C}_{\mathrm{k}}=\{(\mathrm{x}, \mathrm{y}) \mid \varnothing(\mathrm{x}, \mathrm{y}, \mathrm{k})=0\}
$$

Where k represents an artificial time-marching parameter, and then evolves the graph as moves pursuant to the prescribed flow. Therefore, the level set can change topology and expand singularities as remains smooth and preserve the form of a graph.

In this manner, the curve evolution is defined as equation 6.

$$
\partial \mathrm{c} / \partial \mathrm{k}=\mathrm{VN}
$$

Where $\mathrm{V}$ is the speed of curve evolution, $\mathrm{N}$ is the normal vector of inward unit. From $\emptyset\left(\mathrm{C}_{\mathrm{k}}, \mathrm{k}\right)$, the following equation is achieved:

$$
(\partial \emptyset / \partial \mathrm{k})+\nabla \varnothing \bullet(\partial \mathrm{c} / \partial \mathrm{k})=0
$$

According to the level set function definition described above, the vector $\mathrm{N}$ may be written as $\mathrm{N}=-\mathbf{\nabla} \emptyset /\|\mathbf{\nabla} \emptyset\|$. Then it can be implemented corresponding to curve evolution equation (8):

$$
\partial \emptyset / \partial \mathrm{k}=\mathrm{V}\|\boldsymbol{\nabla} \emptyset\|
$$

The initial level set function is generated using the initial given curve. In addition, the function requires to be reinitialized consecutively during the update process which generally takes a lot of computing time.

\section{The Proposed Active Contour Tracking Method}

The presented method seeks a new target location $\mathrm{t} 1$ in the current frame exploiting the k-means procedure and initializing the level set segmentation algorithm from the location $\mathrm{t} 0$ of the target in prior frame. The weighs are computed based on the scale of bandwidth $h$ centered at t0. In other word, the initial curve of each sample is set up and evolved based on the target location 0 obtained by the k-means algorithm. The energy function is refined using the previous knowledge of target model achieved by k-means. The presented active contour tracking algorithm is explained in detail as follow.

\subsection{The Proposed Algorithm}

As described above, k-means clustering algorithm needs $\mathrm{K}$ clusters which should be initialized manually. The number of clusters is 3 in this paper because the skin cancer images are expected to be clustered into less or equal to 3 parts consisting the background (skin), tumor, and possible extra parts. That is, the first cluster is named cluster 1 and the last one is cluster 3 . Since the skin cancer image is gray-scale image in which the minimum intensity value is equal to 0 and the maximum intensity value is equal to 255 , the centroid of cluster 1 is 0 and the centroid of cluster 3 is 255. Equation 9 indicates the calculation of centroid in cluster $\mathrm{k}, \mathrm{C}_{\mathrm{k}}$

$\mathrm{C}_{\mathrm{k}}=\operatorname{rand}^{*} 255 \quad(\mathrm{k}=>1$ and $\mathrm{k}<=3)$

\section{Input: The original skin cancer image Output: Initial Segmented image}

1. Initialize centroid of clusters randomly 2. loop while CTr=CTr-1
If $C \operatorname{Tr}=C \operatorname{Tr}-1$, then disk, $i=\left|C T k-I_{i j}\right|$ for all pixels Assign pixels to the closest distance
Estimate the new centroid for 3 clusters $c t k=A v g\left(I_{k}\right)$

End loop

3. The segmented skin lesion is achieved at location $t 1$ with the scale of $h$

CTr: Cluster centroid at round $r$

$I_{i j}$ : Pixel in an image

Algorithm 1.K-means clustering with 3 clusters 
Algorithm 1 shows the total procedure of k-means clustering of this paper. After determining the initial centroid values of the clusters, the pixels are distributed to one of 3 clusters which the centroid value is the most closest to that pixel intensity. The centroids of clusters are updated by the mean intensity values of its pixels. This process is repeated till reaching the constant value. The target segmented location t1 achieved by this algorithm is used to initialize the level set algorithm. This segmented result is converted to binary for feeding the level set algorithm.

For each sample $t_{i}{ }^{k}$ in level set framework, we initialize a curve by a target segmented location $t 1$ with the scale of $h$ achieved by k-means segmentation algorithm. Hence we evolve the curve using level set algorithm upon the time

$\mathrm{k}, \mathrm{I}_{\mathrm{k}}$, and the target model $\mathrm{t}$ :

$$
\mathrm{C}_{\mathrm{k}}{ }^{1}=\operatorname{evo}\left(\mathrm{s}_{\mathrm{k}}{ }^{1}, \mathrm{I}_{\mathrm{k}}, \mathrm{t}\right)=\mathrm{S}_{\mathrm{k}}{ }^{1}(\mathrm{M})
$$

Where $S_{k}$ indicates the contour at time $k$ and iterates $M$ times to the direction in which the energy function Eimg is reduced

$$
\mathrm{E}_{\mathrm{img}}=\mathrm{E}_{\mathrm{R}}(\mathrm{c} 1, \mathrm{c} 2, \partial)
$$

Where $\mathrm{c} 1, \mathrm{c} 2$ indicate positive constant, $\partial$ is the indicator of regions $(\partial=1$ lesion, $\partial=-1$ background)

By the end of this process, the true target segmentation is achieved which includes the energy smaller than other samples in evolution process. Therefore, the algorithm of this process is illustrated in Algorithm 2.

Input: target segmented location tlwith a scale of $h$
achieved by k-means segmentation algorithm
Output: True target segmentation t2
1. Initialize a curve by a target segmented location t1
2. Run curve evolution in M iterations toward the direction
of energy reduction
3. Calculate the weights
4. If the $(t 2-t 1)<\varepsilon$ and mark $t 2$ as the result

Algorithm 2. Level set segmentation algorithm initializing by k-means clustering algorithm

\section{Experiment Result}

In this section, the proposed method is tested on 40 images taken from digital cameras In order to demonstrate our improved method, multiple algorithms have been run upon the same condition. The first algorithm is the traditional level set (TLS), proposed in [38], the second is modified fuzzy c-means clustering (FCM) [39] in two cut off ( $\mathrm{sw}=0$, cut between the small and middle class, $\mathrm{sw}=1$, cut between the middle and large class), and the last one is the proposed method of this paper.

The original images are initially segmented into 3 clusters for accurate evaluation of lesion area in the image. As this image is used as the curve evolution of level set algorithm, it should be converted to binary image and used to feed the level set algorithm. Figure 1 shows the segmented results achieved by our proposed method (k-means-level set
(KLS)).

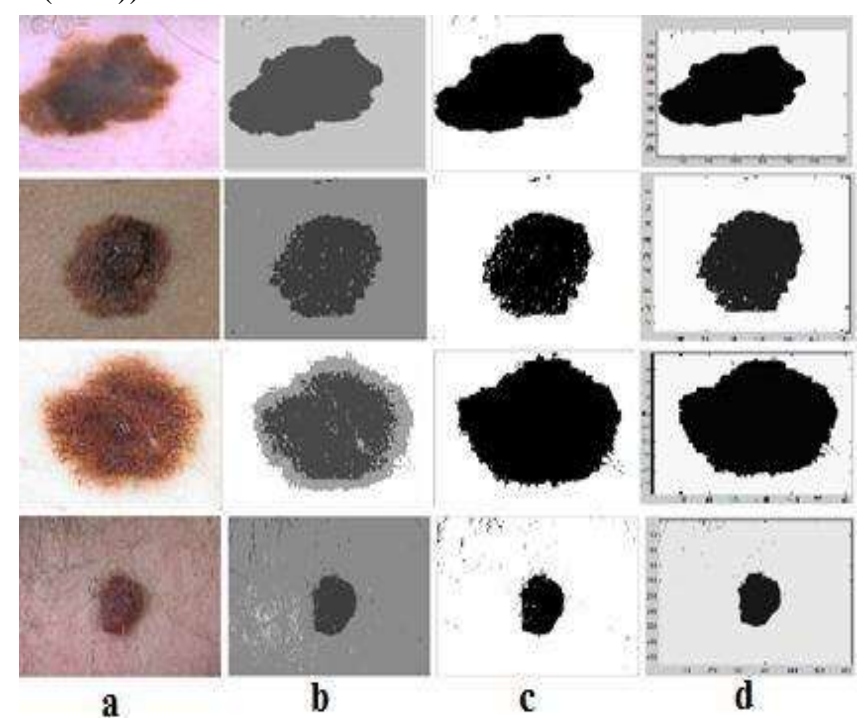

Figure 1. a) Original Image b) Initial k-means segmentation c) Converting the segmented image to binary d) Segmented result of our proposed algorithm after initializing the level set segmentation technique by k-means algorithm

To perform the comparison of our segmented results with the ground truth images which have been segmented manually by the experts, let SR and GT indicate the result of automatic segmentation method and the ground truth segmentation, respectively. Figure 2 indicates this comparison.

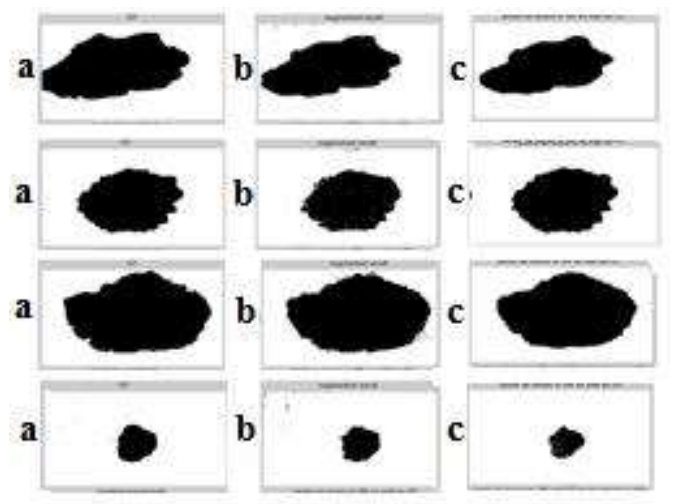

Figure 2. Comparison of ground truth image with the segmented image by proposed algorithm a) Ground truth image b) Segmented result c) Pixels in segmented lesion as well as ground truth (Subscription of "a", "b" images)

Figure 3 demonstrates the results achieved by "modified fuzzy c-means clustering" method which has been applied to compare with our proposed method. 


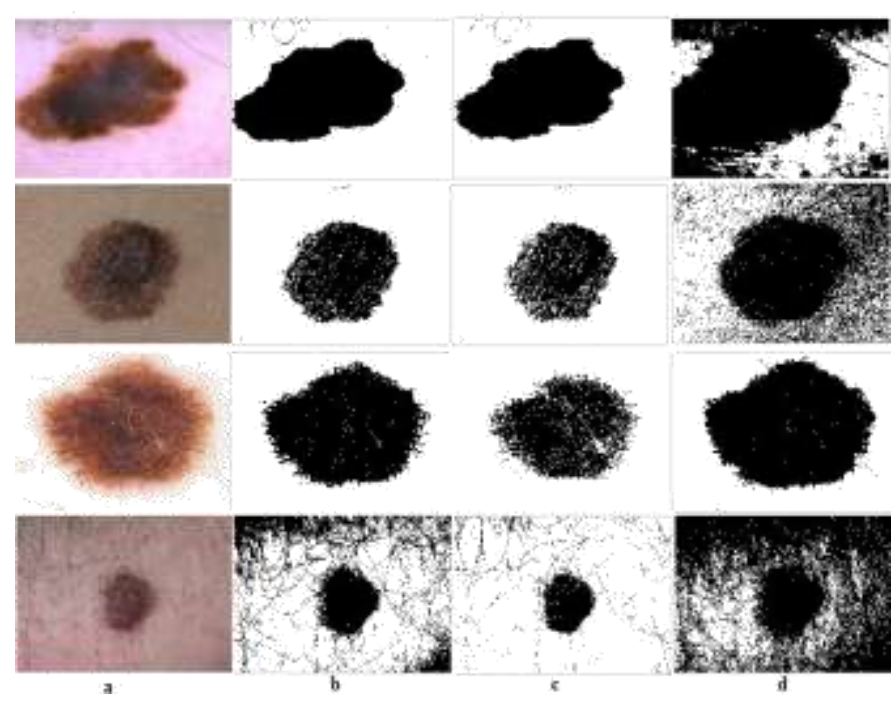

Figure 3. Modified fuzzy c-means clustering in two cut-off position a) Original image b) Otsu thresholding c)

FCM (sw=0) d) FCM (sw=1)

Figure 4 shows the comparison which has been done between our proposed method (KLS), TLS, FCM (sw=0) and FCM (sw=1).

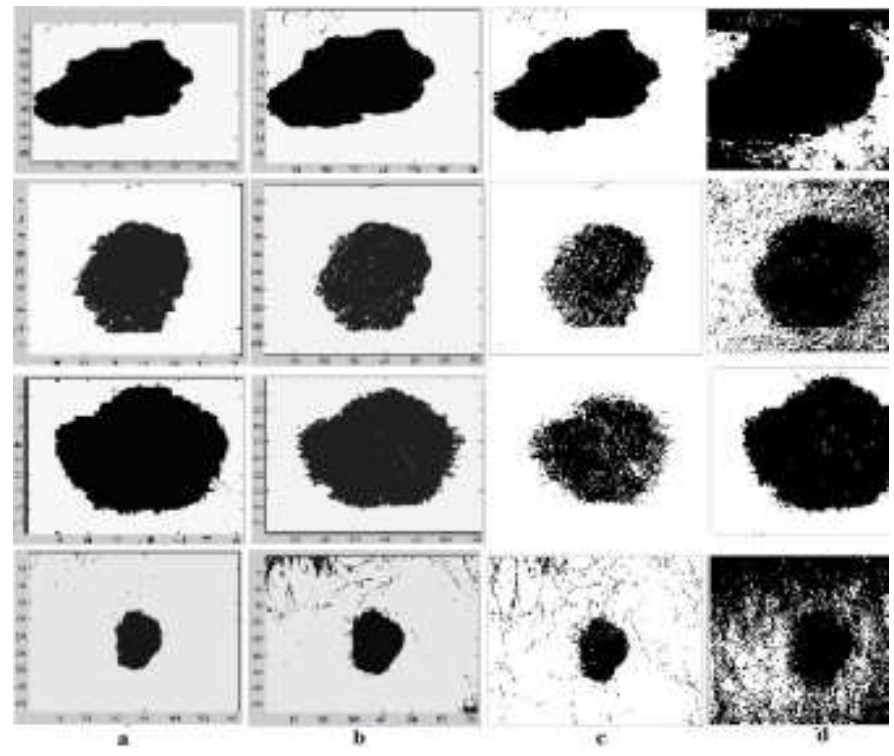

Figure 4.Comparison of a) proposed segmentation method b) traditional level set method c) modified fuzzy c-means clustering $(\mathrm{sw}=0) \mathrm{d}$ ) modified fuzzy c-means clustering $(\mathrm{sw}=1)$

Four different metrics of Border error, Similarity, Hammoude distance and Rms error are used to quantify the boundary differences. Each metric is calculated between the segmented results achieved by TLS, KLS, FCT (sw=0) and FCT ( $\mathrm{sw}=1$ ) with ground truth images (manually segmented images by experts). Table1 shows the statistical comparison of algorithms by these metrics. As it is obvious from table 1, the average border error, Hammoude distance and Rms error between the ground truth and segmented images in KLS is the lowest and similarity is the highest among others. It shows the better performance of KLS than others.

Table 1

Comparison of Proposed method, TLS, FCT ( $w=0)$, FCT $(w=1)$ by the Border error, Similarity, Hammoude distance and Rms error

\begin{tabular}{|l|l|l|l|l|}
\hline & TLS & KLS & $\begin{array}{l}\text { FCT } \\
(\mathrm{sw}=0)\end{array}$ & $\begin{array}{l}\text { FCT } \\
(\mathrm{sw}=1)\end{array}$ \\
\hline Border error & 0.176912 & 0.075871 & 0.124453 & 0.550871 \\
\hline Similarity & 0.810429 & 0.953229 & 0.892076 & 0.458453 \\
\hline $\begin{array}{l}\text { Hammoude } \\
\text { distance }\end{array}$ & 0.447771 & 0.301471 & 0.391818 & 0.676335 \\
\hline Rms Error & 0.335165 & 0.198329 & 0.278412 & 0.656047 \\
\hline
\end{tabular}

For detecting whether our proposed method has statistically significant difference from others, one-way analysis of variance (ANOVA) as a statistical inference is applied with a .05 significance level. It is known as a popular and powerful tool which is robust to nonhomogeneity of the data [40]. The anova test will compare all forty border errors between TLS segmentation results and ground truth with all forty border errors between KLS segmentation results and ground truth with all forty border errors between FCT $(\mathrm{sw}=0)$ segmentation results and ground truth and finally with all forty border errors between FCT $(\mathrm{sw}=1)$ segmentation results and ground truth. The purpose is to find out how significant is the results of our method as compared to other methods. In other word, determine if our method has significantly decreased the border error as compared with other methods. The same process is performed for other three metrics as well. Table 2, which demonstrate One- way ANOVA results of the skin cancer image data, give evidence to the results achieved above in table 1. It represents mean values of Border error, Similarity, Hammoude distance and Rms error metrics on forty experiments by KLS and TLS, KLS and FCT $(\mathrm{sw}=0)$, and KLS and FCT $(\mathrm{sw}=1)$ achieved by 1 -way anova test. As it can be seen in table 2 on the first column, all the mean values are less than 0.05 , which shows the significant difference between KLS than TLS. In second column mean values of Rms error shows the significant difference between KLS and FCT $(\mathrm{sw}=0)$, while the other metrics shows although there are improvement (according to table 1), it is not significant. The mean values of third column show the significant difference between KLS and FCT $(\mathrm{sw}=1)$. 
Table 2

Anova test on Border error, Similarity, Hammoude distance and Rms error between the "proposed method and TLS", "KLS and FCT $(\mathrm{w}=0)$ ", "KLS and FCT $(\mathrm{w}=1)$ "

\begin{tabular}{|l|c|c|c|}
\hline & KLS , TLS & $\begin{array}{l}\text { KLS , FCT } \\
(\mathrm{sw}=0)\end{array}$ & $\begin{array}{l}\text { KLS, FCT } \\
(\mathrm{sw}=1)\end{array}$ \\
\hline Border error & 0.0073 & 0.1332 & $4.53 \mathrm{E}-10$ \\
\hline Similarity & 0.0068 & 0.0638 & $1.92 \mathrm{E}-10$ \\
\hline $\begin{array}{l}\text { Hammoude } \\
\text { distance }\end{array}$ & 0.0236 & 0.1352 & $5.82 \mathrm{E}-06$ \\
\hline Rms Error & 0.0211 & 0.0376 & $2.77 \mathrm{E}-10$ \\
\hline
\end{tabular}

The figure 5 shows the comparative view and difference of
Border error, Similarity, Hammoude distance and Rms error by KLS and TLS, KLS and FCM ( $\mathrm{sw}=0)$, and KLS and FCM $(\mathrm{sw}=1)$ have been achieved by Anova test.

As noticed before, another contribution of this paper achieved is computational time which significantly reduced in compare with TLS method. Table 3 indicates the comparison results.

Table 3

Comparison of Elapsed time between proposed method and TLS

\begin{tabular}{|l|l|l|}
\hline & TLS & KLS \\
\hline Computational time & 0.731007 & 0.716403 \\
\hline
\end{tabular}

Figure 5. Difference of Border error, Similarity, Hammoude distance and Rms error of KLS and TLS, KLS and FCM (sw=0), and KLS and FCM ( $\mathrm{sw}=1)$

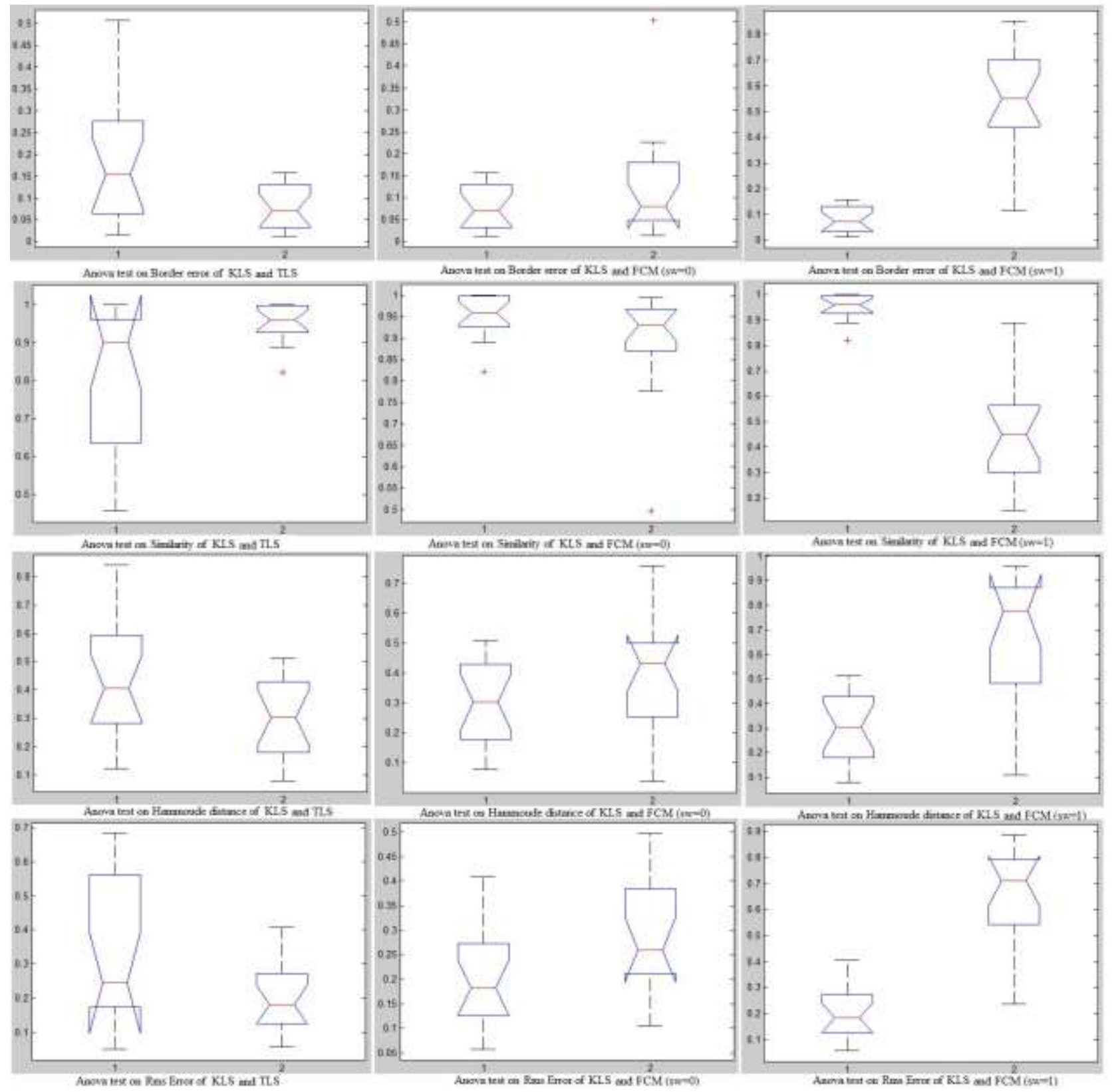


Table 3

Anova test on Elapsed time of KLS and TLS

\begin{tabular}{|l|l|}
\hline & KLS, TLS \\
\hline Computational time & 0.0384 \\
\hline
\end{tabular}

The figure 6 shows the difference of computational cost between KLS and TLS.

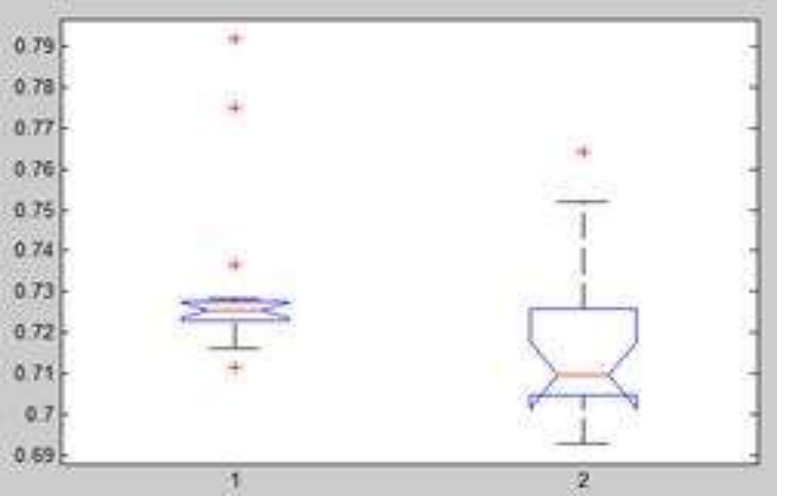

Figure 6.Comparison of computational cost of KLS and TLS by Anova test

Using a joint algorithm, we indicated that the combination of k-means and level set for skin cancer segmentation outperforms the commonly used classification methods such as level set and modified fuzzy c-means clustering, in two cut off ( $\mathrm{sw}=0, \mathrm{sw}=1)$, in terms of accuracy.

\section{Conclusion}

The structuring of an effective segmentation for medical expert systems to assist medical doctors is the purpose of this research. The joint k-means-level set algorithm is proposed. The algorithm employs the k-means segmentation result to initialize level set clustering technique to improve the segmentation results. The precursory along with the conclusive results are much stimulating. As the reader may note, this segmentation architecture is being proposed in such a way that assures the accuracy and effectiveness on its results. In this work, the images data and pathologists ' interaction helped a lot to achieve a good system performance. The experiments have been performed on forty images to evaluate the efficiency of proposed algorithm. Four metrics of Border error, Similarity, Hammoude distance and Rms error were used for this purpose. The promoted results show the successful performance of our proposed method when compared to traditional level set segmentation method and modified fuzzy c-means clustering, in two cut off $(\mathrm{sw}=0$, cut between the small and middle class, $\mathrm{sw}=1$, cut between the middle and large class). To approve our improved results, ANOVA test has been applied for assurance. Additionally, the proposed algorithm can easily be retargeted to apply in other domains of interest.

\section{References}

[1] M. Cheriet, J. N. Said, and C. Y. Suen, "A recursive thresholding technique for image segmentation," IEEE

Transactions on Image Processing, vol. 7, no. 6, pp. 918921, 1998.

[2] S. K. Warfield, K. Michael, F. A. Jolesz, and K. Ron, "Adaptive, template moderated, spatially varying statistical classification," Medical Image Analysis, vol. 4, no. 1, pp. 43-55, 2000.

[3] M. Lalonde, M. Beaulieu, and L. Gagnon, "Fast and robust optic disc detection using pyramidal decomposition and Hausdorff-based template matching," IEEE

Transactions on Medical Imaging, vol. 21, no. 11, pp. 1193-1200, 2001.

[4]N. A. Mat-Isa, M. Y. Mashor, and N. H. Othman, "Automatic seed based region growing for pap smear image segmentation," in Kuala Lumpur International

Conference on Biomedical Engineering. 2002. Kuala Lumpur, Malaysia.

[5]J. K. Paik, Y. C. Park, and S. W. Park, "An edge detection approach to digital image stabilization based on tri-state adaptive linear neurons," IEEE Transactions on Consumer Electronics, vol. 37, no. 3, pp. 521-530, 1991.

[6] X. Yang, Z. Weidong, C. Yufei, and F. Xin., "Image segmentation with a fuzzy clustering algorithm based on Ant-Tree," Signal Processing, vol. 88, no. 10, pp. 24532462, 2008.

[7] S. K. Singh, K. Shishir, G. S. Tomar, K. Ravi, and G. K. A. Santhalia,"Modified framework of a clustering algorithm for image processing applications," in First

Asia International Conference on Modelling \& Simulation, AMS '07, 2007.

[8]F. Hoeppner, "Fuzzy shell clustering algorithms in image processing: fuzzy C-rectangular and 2-rectangular shells," IEEE Transactions on Fuzzy Systems,vol. 5, no.

4, pp. 599-613, 1997.

[9]T. Abeel, Y. V. d. Peer, and Y. Saeys, "Java-ML: A machine learning library," Journal of Machine Learning Research, vol. 10, pp. 931-934, 2009.

[10] A. Y. Al-Omary, and M. S. Jamil, "A new approach of clustering based machine-learning algorithm," Knowledge-Based Systems, vol.19, no.4, pp.248-258, 2006.

[11]P. C. Boutros, and A. B. Okey, "Unsupervised pattern recognition: An introduction to the whys and wherefores of clustering microarray data,"Briefings In

Bioinformatics,vol. 6, no. 4, pp. 331-343, 2005.

[12]J. P. Jesan, "The neural approach to pattern recognition," Ubiquity, vol.5, no.7, 2004.

[13]T. Kanungo, D. M. Mount, N. S. Netanyahu, C. D.

Piatko, R. Silverman, and A. Y. Wu, "An efficient kmeans clustering algorithm: analysis and implementation," IEEE Transactions on Pattern Analysis and Machine Intelligence, vol. 24, no. 7, pp. 881-892, 2002.

[14]H. Wang, W. Wang, J. Yang, and P. S. Yu, "Clustering by pattern similarity in large data sets," in 
Proceedings of the 2002 ACM SIGMOD International Conference on Management of data. 2002, Madison, Wisconsin.

[15] T. Zhang, R. Ramakrishnan, and M. Livny, "BIRCH: an efficient data clustering method for very large databases," in Proceedings of the 1996 ACM SIGMOD international conference on Management of data, 1996, Montreal, Quebec, Canada.

[16] C. Ordonez, "Clustering binary data streams with kmeans," in Proceedings of the 8th ACM SIGMOD workshop on Research issues in data mining and knowledge discovery. 2003, San Diego, California.

[17]D. Auber, and M. Delest, "A clustering algorithm for huge trees," Advances in Applied Mathematics, vol. 31, no. 1 pp. 46-60 2003.

[18]A. S. Mahani, A. E. Carlsson, and R. Wessel,

"Motion repulsion arises from stimulus statistics when analyzed with a clustering algorithm," Biological

Cybernetics, vol. 92, no. 4, pp. 288-291, 2005.

[19]L. Li, P. Ross, M. Kruusmaa, X. Zheng," A

Comparative Study of Ultrasound Image Segmentation Algorithms for Segmenting Kidney Tumors", ISABEL '11,ACM, October 26-29, Barcelona, Spain.

[20]S. Na, L. Xumin, G. yong," Research on k-means Clustering Algorithm", Third International Symposium on Intelligent Information Technology and Security Informatics, 2010.

[21] M. Kamali, G. Samei, "Border preserving skin lesion segmentation", in Proceedings of SPIE 6915, Medical Imaging 2008: Computer-Aided Diagnosis, 2008.

[22]K. Ahmed, T. Jesmin, Md. Zamilur Rahman," Early Prevention and Detection of Skin Cancer Risk using Data Mining", International Journal of Computer Applications (0975 - 8887) Volume 62- No.4, January 2013.

[23]G.Subramanya Nayak, Ottolina Davide, Puttamadappa C," Classification of Bio Optical signals using K- Means Clustering for Detection of Skin Pathology", International Journal of Computer Applications (0975 - 8887) Volume 1 - No. 2, 2010.

[24] R. T. Whitaker." Volumetric deformable models: Active blobs. In Visualization in Biomedical Computing" (1994), pp. 122-134.

[25]A. Masood, A. A. Al-Jumaily, "Fuzzy C mean Thresholding based Level Set for Automated

Segmentation of Skin Lesions", Journal of Signal and Information Processing, Vol. 4, No. 3B, ISSN: 21594465, August 2013, pp. 66-71.

[26] A. Lefohn, J. E.Cates, R. T .Whitaker. "Interactive, GPU-based level sets for 3D segmentation". In Proc. Of Medical Image Computing and Computer Assisted Intervention (MICCAI ${ }^{\text {ce}} 03$ (2003), pp. 564-572.

[27] J. Cates, A. Lefohn, and R. Whitaker,"GIST: an interactive, GPU-based level set segmentation tool for 3D medical images". Medical Image Analysis 8, 3 (2004), 217-231.
[28]Li, B.N., et al.: Integrating spatial fuzzy clustering with level set methods for automated medical image segmentation. Computers in Biology and Medicine 41, 110 (2011)

[29] Q. Abbas, I. Fondón, M. Rashid. Unsupervised skin lesions border detection via two-dimensional image analysis. Computer Methods and Programs in Biomedicine 104, 1-15 (2011).

[30]M M Puranik, S Krishnan," Segmentation of Image Using Watershed and Fast Level set methods",

International Conference and Workshop on Emerging Trends in Technology (ICWET 2011) - TCET, Mumbai, India.

[31]B. Chen, C. Tai Phang, R. Harrison and Y. Pan," Novel Hybrid Hierarchical-k-means Clustering Method (H-k-means) for Microarray Analysis", Workshops and

Poster Abstracts, IEEE Computational Systems Bioinformatics Conference, 2005.

[32] M. Silveira , "Comparison of Segmentation Methods for Melanoma Diagnosis in Dermoscopy Images," IEEE Journal of Selected Topics in Signal Processing, Vol. 3, pp. 35-45, 2009.

[33]M. Naresh Babu, Vamsi K. Madasu, M. Hanmandlu, S. Vasikarla," Histo-pathological Image Analysis using OS-FCM and Level Sets", 39th IEEE Applied Imagery Pattern Recognition Workshop (AIPR),2010.

[34]A.Masood, A.A.Al-Jumaily, A.N. Hoshyar, O. Masood , "Automated Segmentation of Skin Lesions: Modified Fuzzy C mean Thresholding Based Level Set Method", 16th International Multi Topic Conference (INMIC), 2013.

[35] J. MacQueen, Some methods for classification and analysis of multivariate observations. Proc: 5th Berkeley Symp. Math. Statist, Prob, 1:218-297, 1967.

[36]N. Hema Rajini, R.Bhavani," Enhancing K-means and Kernelized Fuzzy C-means Clustering with Cluster Center Initialization in Segmenting MRI brain images",

2011 3rd International Conference on Eectronics Computer Technology(ICECT),2011.

[37] S.J. Osher, J.A. Sethian: Fronts propagation with curvature dependent speed: Algorithms based on hamilton-jacobi formulations. Journal of Computational Physics, 12-49 (1988).

[38] J.A. Sethian: Level set Methods and Fast Marching Methods, 2nd edn. Cambridge University Press, Cambridge (1999).

[39]N. i. jabbar, and S. I. jabbar," A Modified Fuzzy CMeans for Medical Image Thresholding”, Asia Pacific Conference on Environmental Science and Technology Advances in Biomedical Engineering, Vol.6, 2012.

[40]M. Marin-Galiano, J. Kunert," Comparison of ANOVA with the Tobit model for analysing sensory data", Food Quality and Preference 17 (2006) 2 